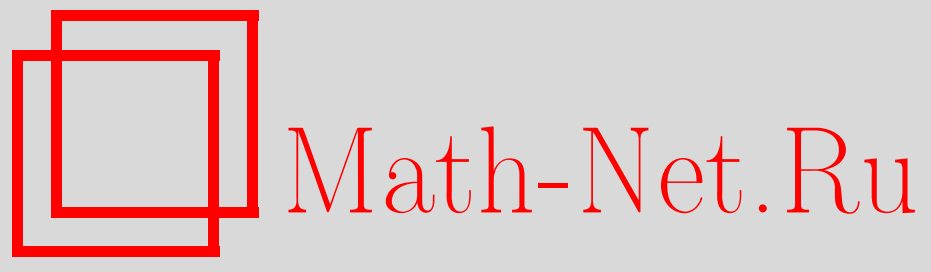

М. В. Невский, Об осевых диаметрах выпуклого тела, Матем. заметки, 2011, том 90, выпуск 2, 313-315

DOI: https://doi.org/10.4213/mzm9201

Использование Общероссийского математического портала Math-Net.Ru подразумевает, что вы прочитали и согласны с пользовательским соглашением http://www . mathnet.ru/rus/agreement

Параметры загрузки:

IP : 54.197 .130 .99

26 апреля 2023 г., 14:48:23

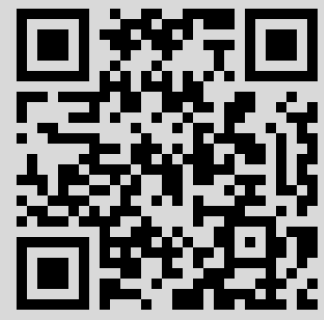




\section{Об осевых диаметрах выпуклого тела}

\section{М. В. Невский}

1. Введение. Пусть $n \in \mathbb{N}$. Элемент $x \in \mathbb{R}^{n}$ будем записывать в виде $x=\left(x_{1}, \ldots, x_{n}\right)$. Через $e_{1}, \ldots, e_{n}$ обозначим канонический базис $\mathbb{R}^{n}$. Положим $Q_{n}:=[0,1]^{n}$.

Пусть $C$ - выпуклое тело в $\mathbb{R}^{n}$, т.е. компактное выпуклое подмножество $\mathbb{R}^{n}$ с непустой внутренностью. Через $\tau C$ обозначим результат гомотетии $C$ относительно центра тяжести с коэффициентом $\tau$. Под транслятом ниже понимается результат параллельного переноса. Для $i=1, \ldots, n$ введем в рассмотрение максимальную длину $d_{i}(C)$ отрезка, содержащегося в $C$ и параллельного оси $x_{i}$. Величину $d_{i}(C)$ будем называть $i$-м осевым диаметром $C$. Понятие осевого или, иначе говоря, аксиального диаметра выпуклого тела было введено Скоттом [1], [2]. Из его результата [1; теорема 1] следует, что если в $C$ можно вписать транслят куба $Q_{n}$, то

$$
\sum_{i=1}^{n} \frac{1}{d_{i}(C)} \geqslant 1 .
$$

Доказательство, приведенное в [1], опирается на $n$-кратное применение к $C$ симметризаций Штейнера. При этом существенно используется то, что все вершины указанного транслята принадлежат границе $C$. В настоящей заметке мы докажем неравенство (1) в более общей ситуации, когда подход работы [1] неэффективен. Как следствие мы получим, что для осевых диаметров любого выпуклого тела $C \subset \mathbb{R}^{n}$ выполняется неравенство

$$
\sum_{i=1}^{n} \frac{1}{d_{i}(C)} \geqslant \alpha(C) .
$$

Здесь и далее символ $\alpha(C)$ обозначает минимальное $\tau>0$, для которого некоторый транслят $\tau C$ содержит $Q_{n}$.

\section{2. Основные результаты.}

Tеорема 1. Пусть для выпуклого тела $C$

$$
\sigma:=\left(\sum_{i=1}^{n} \frac{1}{d_{i}(C)}\right)^{-1} .
$$

Тогда $C$ содержит транслят куба $\sigma Q_{n}$.

ДоказАтЕЛьство. Для $i=1, \ldots, n$ обозначим через $D_{i}$ произвольный отрезок, принадлежащий $C$, параллельный $i$-й координатной оси и имеющий длину $d_{i}:=d_{i}(C)$. Рассмотрим множество $V:=\operatorname{conv}\left(D_{1}, \ldots, D_{n}\right)$. Пусть $\mu_{i}:=\sigma / d_{i}, m^{(i)}$ - центр $D_{i}$. Положим $m:=\sum_{i=1}^{n} \mu_{i} m^{(i)}$. Так как

$$
\mu_{i}>0, \quad \sum_{i=1}^{n} \mu_{i}=\sigma \sum_{i=1}^{n} \frac{1}{d_{i}}=1,
$$

то $m$ есть выпуклая комбинация точек $V$. Поэтому $m \in V$. Обозначим через $Q$ транслят куба $\sigma Q_{n}$, центр которого совпадает с $m$. Покажем, что $Q \subset V$. Произвольная вершина $Q$ имеет вид

$$
v=m+\left( \pm \frac{\sigma}{2}, \ldots, \pm \frac{\sigma}{2}\right)
$$

(C) М. В. Невский, 2011 
с некоторым сочетанием знаков \pm . Имеем

$$
\begin{aligned}
v & =\sum_{i=1}^{n} \frac{\sigma}{d_{i}} m^{(i)}+\sigma\left( \pm \frac{1}{2}, \ldots, \pm \frac{1}{2}\right)=\sigma\left[\sum_{i=1}^{n} \frac{1}{d_{i}} m^{(i)}+\sum_{i=1}^{n}\left( \pm \frac{1}{2} e_{i}\right)\right] \\
& =\sigma \sum_{i=1}^{n} \frac{1}{d_{i}}\left(m^{(i)} \pm \frac{d_{i}}{2} e_{i}\right)=\sum_{i=1}^{n} \mu_{i}\left(m^{(i)} \pm \frac{d_{i}}{2} e_{i}\right) .
\end{aligned}
$$

Отрезок $D_{i}$ параллелен $i$-й координатной оси, и его длина равна $d_{i}$, поэтому $m^{(i)} \pm\left(d_{i} / 2\right) e_{i} \in$ $D_{i}$. Точки $m^{(i)} \pm\left(d_{i} / 2\right) e_{i}$ суть концы $D_{i}$. Таким образом, каждая вершина $Q$ есть выпуклая комбинация концов отрезков $D_{1}, \ldots, D_{n}$ и, следовательно, принадлежит $V$. Значит, $Q \subset V$, а так как $V=\operatorname{conv}\left(D_{1}, \ldots, D_{n}\right) \subset C$, то $Q \subset C$. Теорема 1 доказана.

СледСтвие 1. Пусть С содержит некоторый транслят $Q_{n}$ и не содержит никакого транслята $\tau Q_{n}$ при $\tau>1$. Тогда имеет место (1).

Доказательство. Допустим, что для некоторого $C$ выполняется $\sum 1 / d_{i}(C)<1$. Положим $\sigma:=\left(\sum 1 / d_{i}(C)\right)^{-1}$. По предыдущей теореме $C$ содержит транслят куба $\sigma Q_{n}$. Но так как $\sigma>1$, это противоречит условию. Следствие 1 доказано.

СледСтвие 2. Для любого выпуклого тела $C \subset \mathbb{R}^{n}$ справедливо (2).

ДокАзАтЕльство. Из определения $\alpha(\cdot)$ следует, что выпуклое тело $C^{\prime}:=\alpha(C) C$ удовлетворяет условию следствия 1. Для $C^{\prime}$ выполняется неравенство (1), т.е.

$$
\sum_{i=1}^{n} \frac{1}{d_{i}\left(C^{\prime}\right)} \geqslant 1 .
$$

Остается заметить, что $d_{i}\left(C^{\prime}\right)=\alpha(C) d_{i}(C)$, поэтому (3) эквивалентно (2). Это завершает доказательство.

Нетрудно показать, что для любого выпуклого тела $C \subset \mathbb{R}^{n}$ справедлива следующая нижняя оценка $\alpha(C)$ через осевые диаметры $d_{i}(C)$ :

$$
\alpha(C) \geqslant \frac{1}{n} \sum_{i=1}^{n} \frac{1}{d_{i}(C)} .
$$

Равенство в (4) эквивалентно тому, что $C$ есть гомотетическая копия $Q_{n}$.

3. Случай, когда $C$ - симплекс. Пусть $S$ - невырожденный симплекс в $\mathbb{R}^{n}$. Обозначим вершины $S$ через $x^{(j)}=\left(x_{1}^{(j)}, \ldots, x_{n}^{(j)}\right), 1 \leqslant j \leqslant n+1$. Матрица

$$
\mathbf{A}:=\left(\begin{array}{cccc}
x_{1}^{(1)} & \ldots & x_{n}^{(1)} & 1 \\
x_{1}^{(2)} & \ldots & x_{n}^{(2)} & 1 \\
\vdots & \vdots & \vdots & \vdots \\
x_{1}^{(n+1)} & \ldots & x_{n}^{(n+1)} & 1
\end{array}\right)
$$

является невырожденной. Определитель А и объем $S$ связаны соотношением $\operatorname{vol}(S)=$ $|\operatorname{det}(\mathbf{A})| / n$ !. Пусть $\mathbf{A}^{-1}=\left(l_{i j}\right)$.

Введем в рассмотрение величину $\xi(S):=\min \left\{\tau \geqslant 1: Q_{n} \subset \tau S\right\}$. Включение $Q_{n} \subset S$ эквивалентно равенству $\xi(S)=1$. В [3; теорема 3.1] автор показал, что для любого $i=$ $1, \ldots, n$

$$
\frac{1}{d_{i}(S)}=\frac{1}{2} \sum_{j=1}^{n+1}\left|l_{i j}\right| .
$$


С помощью (5) в [3] было установлено неравенство

$$
\sum_{i=1}^{n} \frac{1}{d_{i}(S)} \leqslant \xi(S)
$$

(см. там теорему 4.1 для случая $Q_{n} \not \subset S$ и следствие 4.2 для случая $Q_{n} \subset S$ ). Приведем формулировки утверждений, которые доказываются с помощью соотношений (5)-(6).

Tеорема 2. Неравенство

$$
\sum_{i=1}^{n} \frac{1}{d_{i}(S)} \leqslant 1
$$

эквивалентно тому, что $Q_{n}$ принадлежит трансляту $S$. Равенство

$$
\sum_{i=1}^{n} \frac{1}{d_{i}(S)}=1
$$

справедливо тогда и толъко тогда, когда $Q_{n}$ принадлежит трансляту $S$ и каждая $(n-1)$ мерная грань последнего содержит вершину $Q_{n}$.

Теорема 3. Имеют место равенства

$$
\alpha(S)=\sum_{i=1}^{n} \frac{1}{d_{i}(S)}=\frac{1}{2} \sum_{i=1}^{n} \sum_{j=1}^{n+1}\left|l_{i j}\right| .
$$

Таким образом, если $C$ - невырожденный симплекс в $\mathbb{R}^{n}$, то (2) является равенством. Обратное утверждение верно лишь для $n=1$, когда любое выпуклое тело $C$ есть отрезок и $\alpha(C)=1 / d_{1}(C)$. Если $n \geqslant 2$, то в качестве подходящего $C$, отличного от симплекса, можно взять множество $x_{1} \geqslant 0, \ldots, x_{n} \geqslant 0, n \leqslant \sum x_{i} \leqslant 2 n$. В этом примере $d_{1}(C)=\cdots=$ $d_{n}(C)=n, \alpha(C)=1$, поэтому левая и правая части (2) одинаковы и равны 1.

\section{СПИСОК ЦИТИРОВАННОЙ ЛИТЕРАТУРЫ}

[1] P. R. Scott, Quart. J. Math. Oxford Ser. (2), 36:3 (1985), 359-362. [2] P. R. Scott, Bull. Austral. Math. Soc., 39:3 (1989), 329-333. [3] М. В. Невский, Матем. заметки, 87:4 (2010), $580-593$.

\section{М. В. Невский}

Поступило

Ярославский государственный университет им. П. Г. Демидова

28.12.2010

E-mail: mnevsk@uniyar.ac.ru 